Laser Chem., 1999, Vol. 19, pp. 83-90

Reprints available directly from the publisher Photocopying permitted by license only
(C) 1999 OPA (Overseas Publishers Association) N.V. Published by license under the Harwood Academic Publishers imprint, part of The Gordon and Breach Publishing Group.

\title{
FEMTOSECOND MID-INFRARED SPECTROSCOPY OF HYDROGEN-BONDED LIQUIDS
}

\author{
S. WOUTERSEN*, U. EMMERICHS and H. J. BAKKER \\ FOM-Institute for Atomic and Molecular Physics, Kruislaan 407, \\ 1098 SJ Amsterdam, The Netherlands
}

(Received 29 April 1997)

We perform femtosecond mid-infrared pump-probe spectroscopy on hydrogen-bonded ethanol dissolved in $\mathrm{CCl}_{4}$. We find that upon excitation of the $\mathrm{OH}$-stretching vibration the hydrogen bonds are predissociated on a femtosecond time scale, and that the predissociation time constant depends strongly on the hydrogen-bond strength.

Keywords: Infrared; hydrogen bonding; femtosecond; pump-probe spectroscopy

\section{INTRODUCTION}

Hydrogen bonding is of major importance in chemistry and biology and has been the subject of ongoing research during the last decades [1]. In particular, the hydrogen bonds of alcohols in apolar solution have been the subject of numerous infrared spectroscopic studies. The coupling between the $\mathrm{O}-\mathrm{H}$ stretching mode and the hydrogen bond in alcohol oligomers has been extensively characterized [2, 3], but detailed knowledge about the dynamical aspects of this coupling was lacking until recently. Using picosecond infrared pump-probe spectroscopy, Graener, Ye and Laubereau [4-6] were the first to reveal that excitation of the $\mathrm{O}-\mathrm{H}$ stretching mode of hydrogen-bonded ethanol results in a fast predissociation of the hydrogen bond, followed by a

\footnotetext{
*Corresponding author.
} 
much slower reassociation process [4]. In their study, a determination of the predissociation lifetime was difficult as it was much shorter than the pulse length. Here, we present the first femtosecond mid-infrared pump-probe study of the $\mathrm{O}-\mathrm{H}$ stretching mode of hydrogen-bonded ethanol in $\mathrm{CCl}_{4}$. Our experiments enable an accurate determination of the hydrogen bond predissociation time constant and its excitation frequency dependence, and confirm the model proposed previously [4].

\section{EXPERIMENT}

Our setup consists of a commercial tunable femtosecond Ti:sapphire laser system (Spectra Physics Tsunami oscillator with a Quantronix 4800 amplifier) which delivers $100 \mathrm{fs}$ pulses of $1 \mathrm{~mJ}$ at a repetition rate of $1 \mathrm{kHz}$. These pulses are downconverted in a parametric generation and amplification setup. In this way nearly transform-limited idler pulses $(\Delta \nu \Delta \tau \sim 0.8)$ of $\sim 200 \mathrm{fs}$ and $30 \mu \mathrm{J}$ are generated in the $2.8-$ $3.2 \mu \mathrm{m}\left(3100-3600 \mathrm{~cm}^{-1}\right)$ wavelength region.

The infrared pulses are split into an intense pump pulse $(\sim 20 \mu \mathrm{J})$ that excites a significant fraction of the molecules and a weak delayed probe pulse $(\sim 1 \mu \mathrm{J})$ that monitors the induced transmission changes. In a pump-probe scan the value of the normalized pump-induced transmission change $\Delta T / T_{0}=T / T_{0}-1$ (where $T_{0}$ is the transmission in absence of the pump pulse) is determined as a function of the delay $\tau$ between pump and probe pulses. We study solutions of $\sim 0.4 \mathrm{~mol} \mathrm{dm}^{-3}$ dissolved in $\mathrm{CCl}_{4}$.

\section{RESULTS AND DISCUSSION}

The effect of hydrogen bonding on the $\mathrm{O}-\mathrm{H}$ stretching mode can clearly be seen in Figure 1, which shows the linear infrared absorption spectrum of $1.5 \mathrm{~mol} \mathrm{dm}^{-3}$ solution of ethanol in $\mathrm{CCl}_{4}$. The fundamental $\mathrm{O}-\mathrm{H}$ stretching region contains three distinct bands [4]. The intense broad band centered at $\nu_{\delta} \sim 3330 \mathrm{~cm}^{-1}$ is due to absorption of hydroxylic groups at internal positions of hydrogen-bonded oligomers. The narrow absorption band at $3625 \mathrm{~cm}^{-1}$ is due to both 


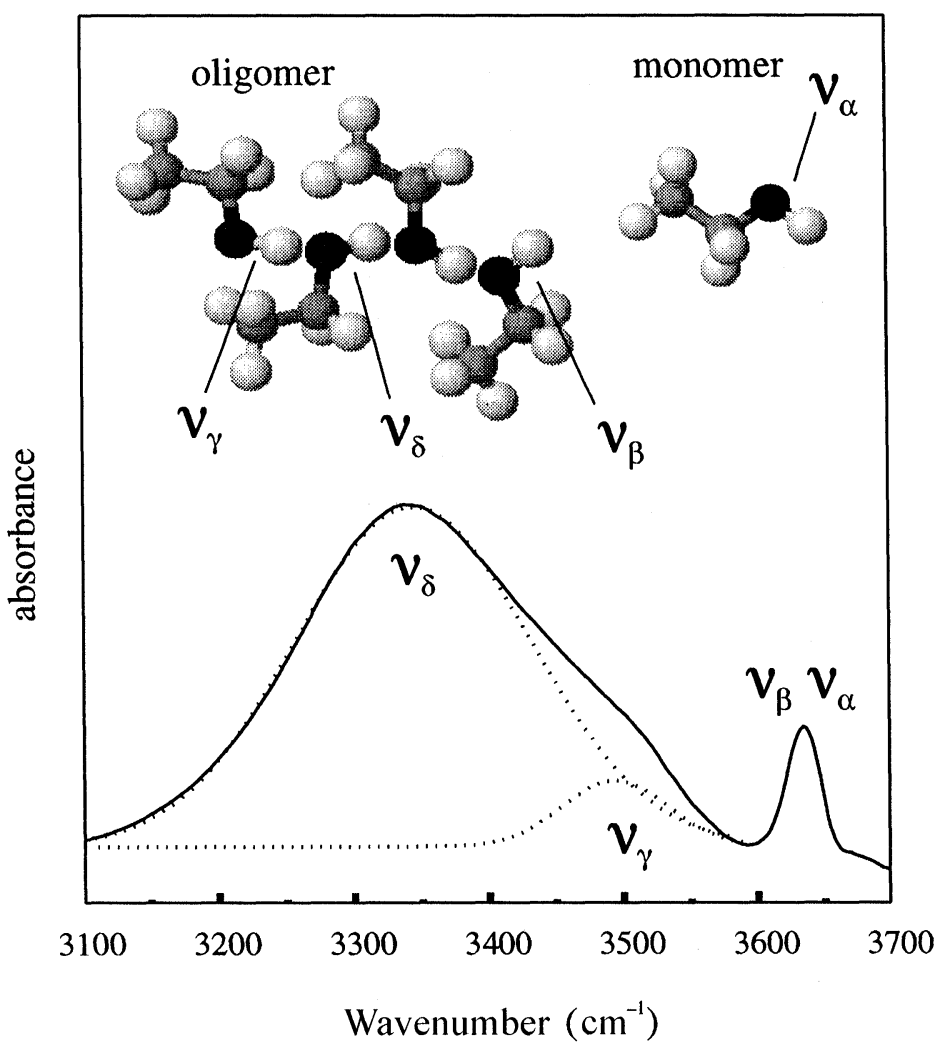

FIGURE 1 Infrared absorption spectrum of $1.5 \mathrm{~mol} \mathrm{dm}^{-3}$ ethanol in $\mathrm{CCl}_{4}$ in the spectral region from 3100 to $3700 \mathrm{~cm}^{-1}$ at room temperature, showing the $\mathrm{O}-\mathrm{H}$ stretching bands, labeled according to Ref. [4]. The dotted curves show the contributions of the internal hydroxylic groups and the terminal hydrogen-bond donor hydroxylic groups, centered at $3330 \mathrm{~cm}^{-1}\left(\nu_{\delta}\right)$ and $3500 \mathrm{~cm}^{-1}\left(\nu_{\gamma}\right)$, respectively. The narrow absorption band at $3625 \mathrm{~cm}^{-1}$ is due to hydroxylic groups of isolated molecules $\left(\nu_{\alpha}\right)$ and of hydrogen-bonded molecules at terminal hydrogen-bond acceptor positions $\left(\nu_{\beta}\right)$.

hydroxylic groups of non-hydrogen bonded ethanol molecules $\left(\nu_{\alpha}=3630 \mathrm{~cm}^{-1}\right)$ and hydrogen-bond acceptor molecules at the end of open chain oligomers $\left(\nu_{\beta}=3620 \mathrm{~cm}^{-1}\right)$. The hydrogen-bond donor end groups of the open chain oligomers absorb at $\nu_{\gamma} \sim 3500 \mathrm{~cm}^{-1}$.

We have recorded pump-probe scans with parallel polarizations of the pump and probe beams at four excitation frequencies within the broad $\mathrm{O}-\mathrm{H}$ stretching band of the hydrogen-bonded ethanol molecules at internal positions. Figure 2 shows a delay scan recorded 


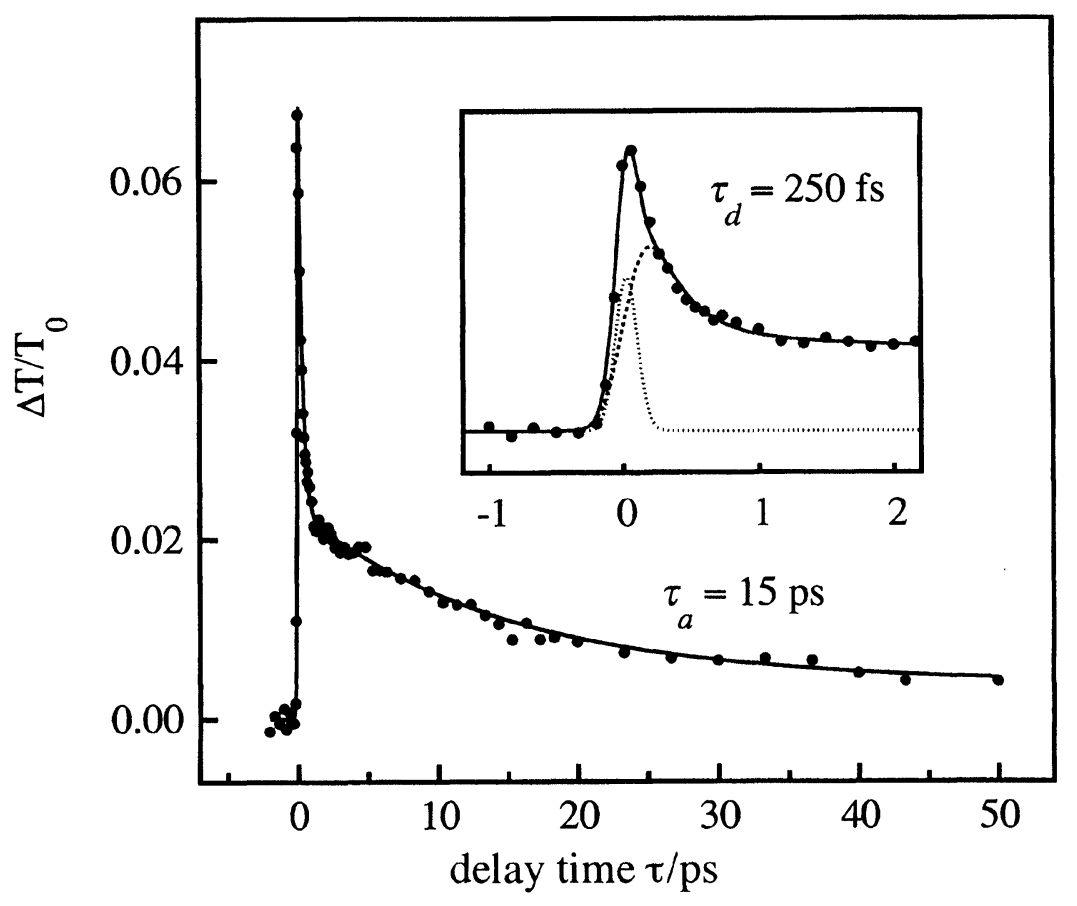

FIGURE 2 Delay scan showing the relative transmission change $\Delta T / T_{0} v s$ delay, recorded at an excitation frequency of $3330 \mathrm{~cm}^{-1}$. The solid curve represents a calculation using values of $\tau_{d}=270 \mathrm{fs}$ and $\tau_{a}=15 \mathrm{ps}$. In the inset, the dashed curve represents the incoherent contribution to the signal, the dotted curve the coherent contribution.

at $3330 \mathrm{~cm}^{-1}$, close to the maximum of the absorption band. The population of the $\nu_{\mathrm{OH}}=1$ state of the hydrogen-bonded ethanol molecules results in a decreased absorption at the $\nu_{\mathrm{OH}}=0 \rightarrow 1$ transition frequency. This bleaching decays in two stages: first a rapid decay takes place (time constant $\sim 250 \mathrm{fs}$ ), followed by a slower decay to a slightly elevated transmission level (time constant $\sim 15 \mathrm{ps}$ ). The observed decay of the bleaching can be explained if the vibrational relaxation takes place by a rapid energy transfer from the $\mathrm{O}-\mathrm{H}$ stretching coordinate $r_{\mathrm{OH}}$ to the hydrogen-bond coordinate $r_{\mathrm{OH}} \ldots \mathrm{O}$ [4, 7]. The excitation energy exceeds the binding energy of the hydrogen bond, which is $\sim 2000 \mathrm{~cm}^{-1}$ [3]. This means that relaxation to the $\nu_{\mathrm{OH}}=0$ state leads to dissociation of the hydrogen bond. The 
depopulation of the $\nu_{\mathrm{OH}}=1$ state leads to a decrease of the population difference with the vibrational ground state and thereby to a decrease of the bleaching. The dissociation alters the distribution of hydroxylic groups, decreasing the number of internal groups and increasing the number of terminal groups. Since the terminal groups absorb at $3500 \mathrm{~cm}^{-1}$ and $3625 \mathrm{~cm}^{-1}$ [4], these will not be observed by the probe at $3330 \mathrm{~cm}^{-1}$, so that the predissociation leads to a decrease of the bleaching to half its initial value. As the system relaxes to thermal equilibrium, the hydrogen bonds are again formed and the residual bleaching vanishes with a time constant of $\sim 15 \mathrm{ps}$. The transmission decays to a plateau value which is somewhat higher than observed for negative delay times because the equilibrium is reached at a slightly elevated temperature, at which there is a relatively smaller concentration of oligomers and thus a reduced absorption at $3330 \mathrm{~cm}^{-1}$.

Figure 3 shows a pump-probe scan recorded at an excitation frequency of $3225 \mathrm{~cm}^{-1}$, at the red side of the absorption band. This scan shows an initial transmission decrease, which vanishes very rapidly (time constant $\sim 250 \mathrm{fs}$ ), followed by a bleaching which decays much more slowly (time constant $\sim 15 \mathrm{ps}$ ). The initial transmission decrease results from absorption of the excited $\nu_{\mathrm{OH}}=1$ state. Overtone studies have shown that the center frequency of the $1 \rightarrow 2$ absorption band is located at $3110 \mathrm{~cm}^{-1}$ [8]. Since the homogeneous linewidth of the $1 \rightarrow 2$ absorption band can be expected to be larger than that of the $0 \rightarrow 1$ transition [9], and the cross section of the $1 \rightarrow 2$ transition is approximately twice that of the $0 \rightarrow 1$ transition, at $3225 \mathrm{~cm}^{-1}$ the net effect of the bleaching at $3330 \mathrm{~cm}^{-1}$ and the induced absorption at $3110 \mathrm{~cm}^{-1}$ is a transmission decrease. The hydrogenbond dissociation results in a residual bleaching at the $\mathrm{O}-\mathrm{H}$ stretching frequency of the hydrogen-bonded internal hydroxylic groups, which causes the bleaching observed in Figure 3. The occurence of this bleaching can only be explained from a breaking of the hydrogen bond. Hence, the observed transient in Figure 3 proves unambiguously that the observed dynamics indeed results from vibrational relaxation and not from spectral diffusion effects. The rapid predissociation implies that spectral diffusion, if any, has to take place within $250 \mathrm{fs}$. As the hydrogen bonds are again formed, the bleaching vanishes. The time constant $\tau_{a}$ of the hydrogen bond 


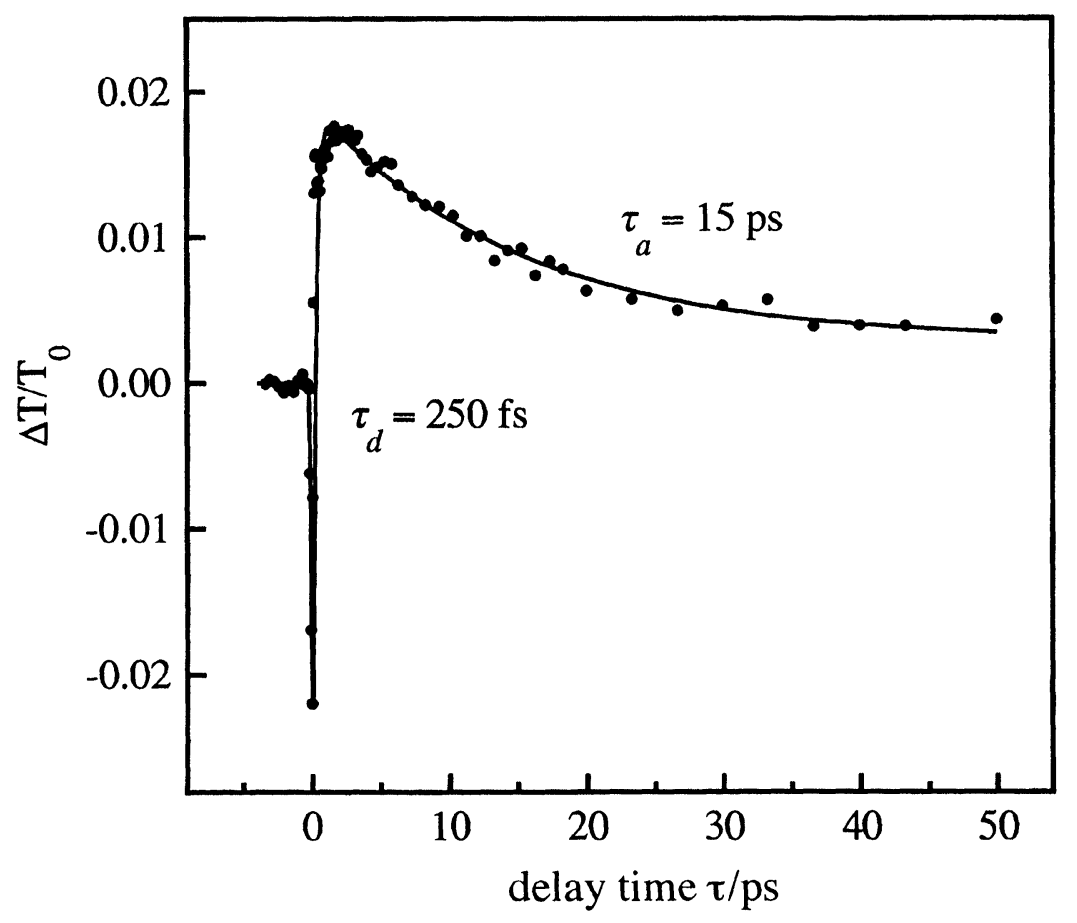

FIGURE 3 Delay scan showing the relative transmission change $\Delta T / T_{0}$ vs delay, recorded at an excitation frequency of $3225 \mathrm{~cm}^{-1}$. The solid curve represents a calculation using values of $\tau_{d}=250 \mathrm{fs}$ and $\tau_{a}=15 \mathrm{ps}$.

reassociation process is the same as observed at $3330 \mathrm{~cm}^{-1}$. The observation of induced $1 \rightarrow 2$ absorption confirms that the system is in the $\nu_{\mathrm{OH}}=1$ state during a finite time before breaking of the hydrogen bond occurs.

To investigate whether the hydrogen-bond predissociation rate varies over the absorption band, we have also performed pump-probe scans at excitation frequencies of 3390 and $3450 \mathrm{~cm}^{-1}$ (Fig. 4). It is clearly observed that the predissociation lifetime increases significantly with the excitation frequency.

The numerical analysis of the data, in which coherent couplingeffects were taken fully into account [10], was performed using as input parameters the experimentally determined FWHM of the pulse envelope and of the power spectrum. A least-squares fit was performed 


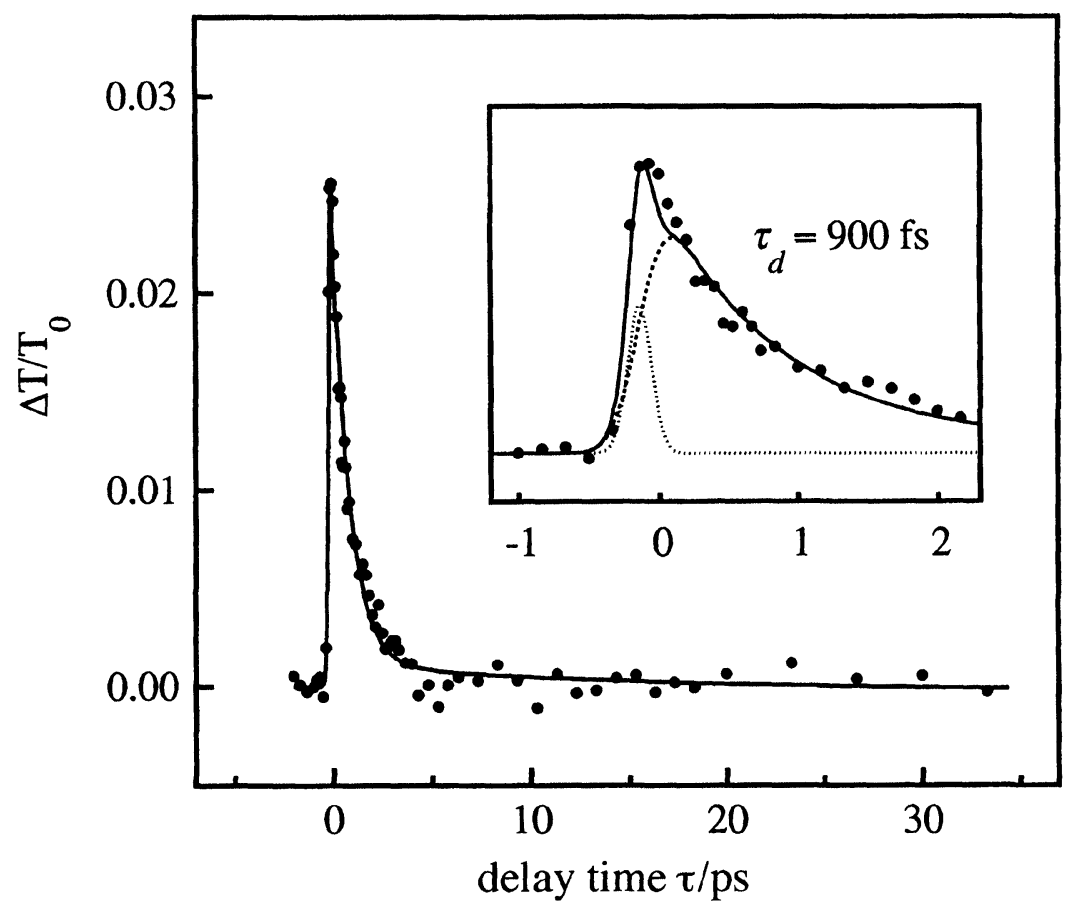

FIGURE 4 Delay scan showing the relative transmission change $\Delta T / T_{0} v s$ delay, recorded at an excitation frequency of $3450 \mathrm{~cm}^{-1}$. The solid curve represents a calculation using a value of $\tau_{d}=870 \mathrm{fs}$ and $\tau_{a}=15 \mathrm{ps}$. In the inset, the dashed curve represents the incoherent contribution to the signal, the dotted curve the coherent contribution.

to obtain values for the predissociation time constant $\tau_{d}$ at the four different excitation frequencies ranging from 3225 to $3450 \mathrm{~cm}^{-1}$. The results of the least-squares fitting procedure are given in Table I and are shown as the solid curves in Figures 2-4. The value obtained for

TABLE I Predissociation lifetime $\tau_{d}$ and reassociation time constant $\tau_{a}$ at different excitation frequencies $\nu$, obtained from numerical analysis of the data (shown as the solid lines in Figs. 2-4). The values in parentheses represent $2 \sigma$

\begin{tabular}{lll}
\hline$\nu / \mathrm{cm}^{-1}$ & $\tau_{d} / f s$ & $\tau_{a} / p s$ \\
\hline 3225 & $250(50)$ & $15(3)$ \\
3330 & $270(40)$ & $15(3)$ \\
3390 & $440(40)$ & \\
3450 & $870(90)$ & \\
\hline
\end{tabular}


the time constant of the hydrogen-bond reassociation process is in agreement with the value of $20 \pm 5 \mathrm{ps}$ obtained in the picosecond studies [4].

The largest observed vibrational lifetime ( $\sim 900 \mathrm{fs})$ of the hydrogenbonded ethanol is still two orders of magnitude shorter than the lifetime of non-hydrogen bonded ethanol in $\mathrm{CCl}_{4}$ solution, which has been found to be $70 \mathrm{ps}$ [11]. This indicates that the hydrogen-bond predissociation is indeed a very efficient relaxation channel. It is evident that the predissociation time constant depends strongly on the excitation frequency. This means that the $\mathrm{O}-\mathrm{H}$ stretching band of the hydrogen-bonded oligomers is inhomogeneously broadened, as was observed previously in picosecond studies on EtOH:CCl [4]. It is well-known that the redshift of the $\mathrm{O}-\mathrm{H}$ stretching frequency is a measure of the hydrogen-bond strength [1]. Apparently, weaker hydrogen-bonding leads to slower predissociation. In fact, studies on hydrogen-bonded acid:base systems [12] have shown that for very weak hydrogen bonds (small redshift) no significant predissociation occurs. To our knowledge, a frequency dependence of the predissociation rate of the hydrogen bond, which has been predicted in theoretical studies [7], has not been observed previously.

\section{References}

[1] The Hydrogen Bond, Edited by Schuster, P., Zundel, G. and Sandorfy, C. (Elsevier, Amsterdam, 1976).

[2] Errera, J. and Mollet, P. (1936). Nature, 138, 882.

[3] Liddel, U. and Becker, E. (1957). Spectrochim. Acta, 10, 79.

[4] Graener, H., Ye, T. Q. and Laubereau, A. (1989). J. Chem. Phys., 90, 3413.

[5] Graener, H., Ye, T. Q. and Laubereau, A. (1989). J. Chem. Phys., 91, 1043.

[6] Graener, H. and Ye, T. Q. (1989). J. Phys. Chem., 93, 5965.

[7] Staib, A. and Hynes, J. T. (1993). Chem. Phys. Lett., 204, 197.

[8] Bourderon, C. and Sandorfy, C. (1973). J. Chem. Phys., 59, 2527.

[9] Graener, H., Ye, T. Q. and Laubereau, A. (1990). Phys. Rev. B, 41, 2597.

[10] Vardeny, Z. and Tauc, J. (1981). Opt. Commun., 39, 396.

[11] Heilweil, E. J., Casassa, M. P., Cavanagh, R. R. and Stephenson, J. C. (1986). J. Chem. Phys., 85, 5004.

[12] Arrivo, S. M. and Heilweil, E. J. (1996). J. Phys. Chem., 100, 11975. 